\title{
Spontaneous Variability and Reactive Postural Beat-to-Beat Changes of Integral ECG Body Surface Potential Maps
}

\author{
E. KELLEROVÁ ${ }^{1}$, V. SZATHMÁRY ${ }^{1}$, G. KOZMANN ${ }^{2,3}$, K. HARASZTI $^{2}$, Z. TARJÁNYI $^{3}$ \\ ${ }^{1}$ Institute of Normal and Pathological Physiology, Slovak Academy of Sciences, Bratislava, \\ Slovakia, ${ }^{2}$ Research Institute for Technical Physics and Materials Science, Hungarian Academy of \\ Sciences, Budapest, Hungary, ${ }^{3}$ University of Pannonia, Veszprém, Hungary
}

Received October 19, 2009

Accepted April 16, 2010

On-line June 9, 2010

\section{Summary}

There is virtually no information on spontaneous variability of ECG body surface potential maps (BSPMs) and on dynamics of their reactive changes in healthy subjects. This study evaluated quantitatively the depolarization (QRS) and repolarization (QRST) parameters derived from the respective integral BSPMs, constructed beat-to-beat, from continual body surface ECG records in 9 healthy men resting supine, during head-up tilting and sitting. Spontaneous variability of the BSPMs parameters, both at rest and during postural reactions, was characterized by significant respiratory and low frequency oscillations, more pronounced when related to repolarization. Head-up tilting and sitting-up evoked significant decrease in the QRST-BSPM amplitudes, widening of the angle $\alpha$ and reduction of nondipolarity indexes, compared to the respective supine values. All these changes were gradual, characterized by transition phenomena and prolonged after-effects. Tilting back to horizontal restored the resting supine values. The postural effects on depolarization were individually more variable and in the average showed a minimal QRS-BSPM amplitude increase. Beat-to-beat analysis of a train of ECG BSPMs provided the first evidence of spontaneous, non-random, respiratory and low frequency oscillations of the ventricular repolarization pattern, and the first insight into the dynamics of body posture associated changes in ventricular recovery.

\section{Key words}

Integral ECG body surface maps - Beat-to-beat variability • Respiratory and low frequency oscillations • Postural effects

\section{Corresponding author}

E. Kellerová, Institute of Normal and Pathological Physiology, SAS, Sienkiewiczova 1, SK 81371 Bratislava 1, Slovak Republic. Fax: + 4212 52968516. E-mail: eva.kellerova@savba.sk

\section{Introduction}

In order to gain detailed information on the cardiac electric field, attention has been focused in the past, on studying the information content of the ECG body surface potential maps (BSPMs). In spite of the fact that this research and the tentative clinical application of mapping culminated more than a decade ago, it still remained an important basic research area for electrocardiography. Several papers offer basic data on the pattern of ventricular depolarization and repolarization time-integral BSPMs in clinically normal subjects at rest (Montague et al. 1981, Sutherland et al. 1983, Green et al. 1985, Kozlíková et al. 1988, Kozmann et al. 1998, Slavíček et al. 2001, Ruttkay-Nedecký and Regecová 2002, Kellerová et al. 2006). However, in subjects with no cardiovascular symptomatology, only a few studies have dealt so far with the problem of spontaneous variations in the pattern, or with reactive changes of BSPMs due to different physiological conditions, involving variations in the autonomic drive of ventricles (Sutherland et al. 1983, Schusterman et al. 1998, Slavíček et al. 1998, Ruttkay-Nedecký and Regecová 2002, Pišvejcová et al. 2002, Kellerová et al. 2006, Žd'árská et al. 2006). In addition, there is a relative 
limitation in interpreting these studies, as only a single BSPM from one selected heartbeat (technical bounds of the equipments), or averages from a short sequence of several maps were used to evaluate the significance of their changes in different situations. Computer-based BSPMs recording (Kozmann 2006, Kozmann and Haraszti 2007) enabled a beat-to-beat analysis of the dynamic behavior of the cardiac electric field.

The aim of the present study was to provide insight into the previously unexplored intra-individual spontaneous BSPMs variability, and to examine beat-tobeat, the reactive changes and transition phenomena in the depolarization and repolarization parameters of the integral BSPMs induced by changing body posture.

\section{Methods}

Nine male volunteers (average age $29 \pm 16$ years, body weight $73 \pm 11 \mathrm{~kg}$, BMI $23 \pm 3$, fat $\% 14.8 \pm 6.8$, abdominal circumference $83 \pm 9 \mathrm{~cm}$ ), with normal cardiac history, physical examination and standard ECG were studied. None of them was smoking or taking any medication.

\section{Study protocol}

Each subject had four continuous BSPM recordings, under spontaneous tidal respiration: i) $300 \mathrm{~s}-$ resting supine, under quiet baseline conditions; ii) passive head up tilting partitioned into $30 \mathrm{~s}$ - starting supine position, $90 \mathrm{~s}-$ gradual tilting to plus $60^{\circ}$, and $180-240 \mathrm{~s}$ - in the passive tilted position; iii) head down tilting partitioned into $30 \mathrm{~s}-$ starting tilted position, $90 \mathrm{~s}-$ gradual head down tilting to horizontal, and 180-240 s supine rest; iv) $180 \mathrm{~s}-$ sitting rest, recorded immediately after active sitting up from the supine position. Depending on the heart rate, 906-1262 BSPMs were evaluated in individual subjects.

The Ethics Committees of the Institutions approved the design of this study and all subjects gave their informed consent.

\section{Data recording and processing}

Using a layout of 64 torso electrodes, unipolar ECG records were obtained by the BioSemi Mark-8 System (Netherlands) (Hoekema et al. 1998, Khaddoumi et al. 2006). Adhesive one-use electrodes were used, to prevent their incidental displacement due to body position changes. The System is also secured by signalizing any electrode detachment. Data processing started with the identification of individual beats, high-precision determination of QRS fiducial points (time instants of the most negative derivative of the QRS waves), and classification of QRS patterns. Subsequently $Q_{o n}, S_{\text {end }}$ and $T_{\text {end }}$ points were manually marked based on the diagram of time-aligned averaged superimposed unipolar leads. The relative immunity of QRS and QRST integral computations on $\pm 5 \mathrm{~ms}$ error in $\mathrm{Q}_{\mathrm{on}}, \mathrm{S}_{\text {end }}$ wave limits, and $\pm 20 \mathrm{~ms}$ errors in $\mathrm{T}_{\text {end }}$ determinations under steady-state conditions has been validated earlier by Kozmann and Haraszti (2007). In transient test conditions (e.g. tilting) for the individual cycle detection a high fidelity fiducial point locator was used, where a strict correlation-based clustering technique (with correlation limit $>0.98$ ) sorted the heart cycles into quasi-stationary subgroups for which the assumption of Kozmann and Haraszti (2007) holds as well. After a linear baseline adjustment of the measured 64 ECG signals, in other 128 chest locations of the regular 192 measuring point arrangement, ECG signals were estimated following the principle and a transfer matrix for the 32 to 192 lead estimations, suggested by Lux et al. (1978). In our approach the same transfer matrix was applied in the unmeasured 128 thoracic points, but the real data were used in the remaining points.

From the resulting dataset, the QRS, ST and QRST integral BSPMs for each heartbeat were constructed in 192 points of a regular grid (16 columns x 12 rows), representing the human chest surface. The corresponding BSPMs are represented on Figure 1. Each contour line on the isointegral map connects points of equal time-integral value, from zero towards the maximum and minimum.

Following parameters were beat-to-beat evaluated: R-R intervals (RR), the values and localizations of maxima (QRSmax or QRSTmax) and minima (QRSmin or QRSTmin) of the respective integral BSPMs, their peak-to-trough amplitudes (QRSampl or QRSTampl), as well as three parameters computed in the $12 \mathrm{D}$ space of Karhunen-Loeve (KL) eigenvectors (Lux et al. 1981) - the angle $\alpha$ between the QRS and QRST eigenvectors and the nondipolarity indices (NDI) characterizing beat-to-beat the topography of the QRS and QRST integral maps (Abildskov et al. 1985).

The semi-automatic oscillometric method (Omron 705 IT) was used to measure the blood pressure (BP) repetitively in each experimental situation. As an estimate of the left ventricular performance the double product (DP) i.e. the product of simultaneous heart rate and systolic BP values was calculated. 


\section{Statistical analysis}

Throughout the study all numeric data within the situations, in the group or intra-individually are presented as means \pm standard deviations (S.D.). Comparison of data was performed by the two-tailed Student's t-test and F-test. Discrete Fourier Transform in the frequency

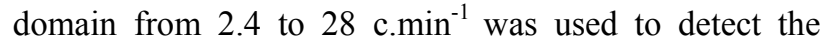
possible periodicity hidden in the variability of the BSPM parameters in the steady-state supine position at the resting heart rate, with sampling in the real $\mathrm{RR}$ intervals. The DC component, if present has been eliminated.

Differences between mean values of evaluated parameters were considered significant at $\mathrm{p}<0.01$.

\section{Results}

Integral depolarization and repolarization BSPMs in continual beat-to-beat records

Because of interference with inter-individual diversity in BSPMs sequences, a detailed analysis of the intra-individual spontaneous BSPMs variability and of their reactive changes was documented in this paper in one and the same healthy subject, and verified by groupmean evaluations.

The somatometric indices of the investigated subjects were more or less in a fair health range. No significant relations between the BSPM parameters and that of body build were found (considering that the group was small, but relatively homogeneous).

The average integral QRS, ST and QRST BSPMs computed from 400 consecutive ECG complexes in one subject supine at rest, and the maps of respective standard deviations are shown in Figure 1. The pattern of all maps is dipolar, with positivity over the left precordium, negative values over the right anterior torso in QRS and right posterior torso in ST and QRST BSPMs (Fig. 1A), but it varies spontaneously in spite of the basal resting conditions. The greatest within subject standard deviations were found in the precordial region of positive potentials, dominating in repolarization ST maps and contributing thereby also to the variability of the QRST BSPM (Fig. 1B).

In the continual follow-up, the pattern of BSPMs clearly oscillates from beat-to-beat. Figure 2A shows recordings of $\mathrm{RR}$, the QRSampl characterizing the ventricular depolarization and the QRSTampl reflecting mainly changes of myocardial recovery processes, NDI measures of the respective maps and of the angle $\alpha$, at rest and in their dynamics during the body-positiondependent cardiovascular reactions in one subject. There are several components of the considerable variability of the investigated BSPMs parameters, which were quantitatively evaluated.
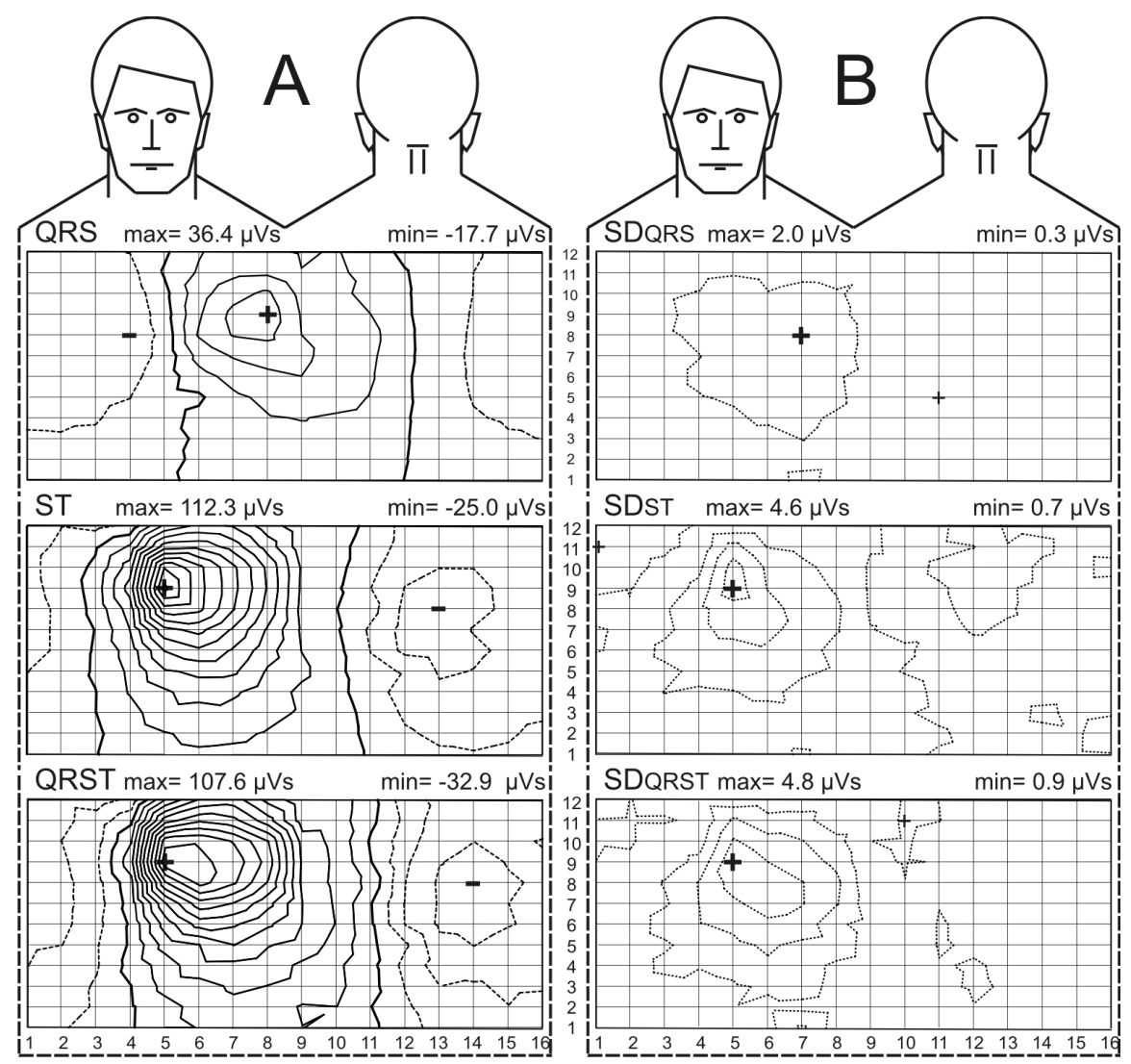

Fig. 1. A. Average $(n=400)$ integral QRS, ST and QRST BSPMs from one subject supine at rest (M.T.). Contours are separated by $10 \mu \mathrm{Vs}$. B. Maps of standard deviations in respective BSPMs. Contours are separated by $1 \mu \mathrm{Vs}$. + site of maximal S.D., + site of minimal S.D. 


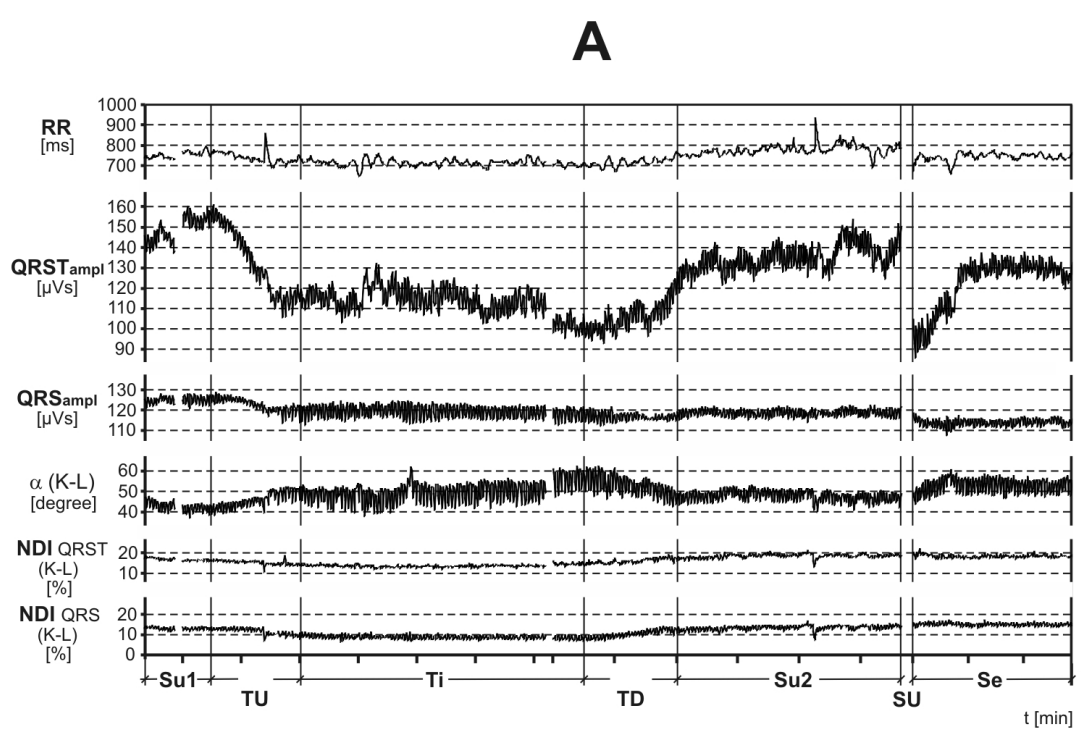

B

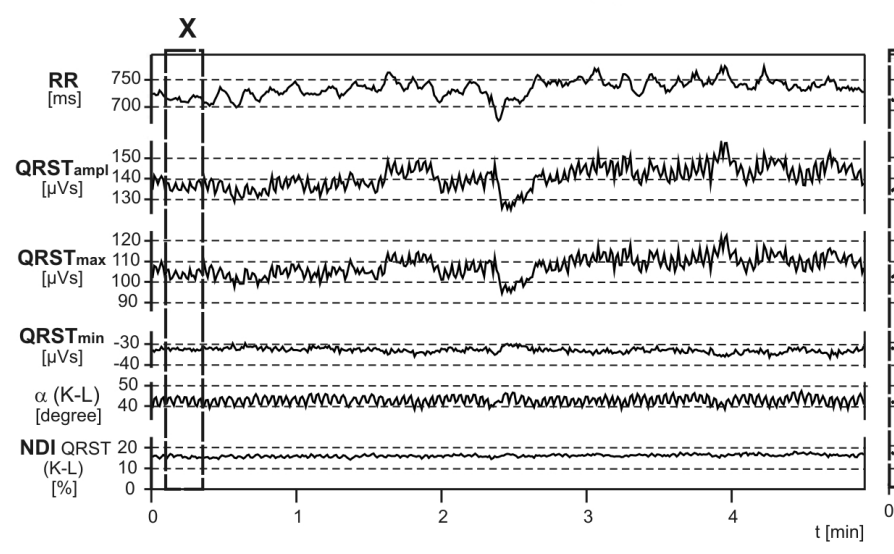

Spontaneous heart rate and BSPM parameters variability

Fluctuations of the values around the mean, visible mainly in RR and QRSTmax, QRSTampl or $\alpha$, present at control rest supine (Fig. 2B), (as well as during stabilized periods of head-up tilted (Ti) or seated (Se) positions, Fig. 2A), when analyzed in their non-random components, provided in this particular subject strikingly different power spectra. In the control rest supine period, during normal tidal breathing (frequency of 18-20 c. $\mathrm{min}^{-1}$ ), the majority of RR variability was represented by predominant low frequency rhythms in the range from $\sim 0.04$ to $0.15 \mathrm{~Hz}\left(2.4-9\right.$ c. $\left.\mathrm{min}^{-1}\right)$, while the respiratory sinus arrhythmia was missing (Fig. 3). On the other hand, in the course of the parameters representing recovery processes in the ventricles, i.e. in QRSTampl, QRSTmax and $\alpha$, illustrated at rest supine (Fig. 2B), two clearly separated periodical components were present: lowfrequency (2.4-9 c. $\left.\mathrm{min}^{-1}\right)$ and high-frequency component ( 0.15 to $0.40 \mathrm{~Hz}$ i.e. $9-24$ c. $\mathrm{min}^{-1}$ ) (Fig. 3).

Similar pattern of BSPMs variability at rest in supine position, with rhythmic oscillations in the LF and

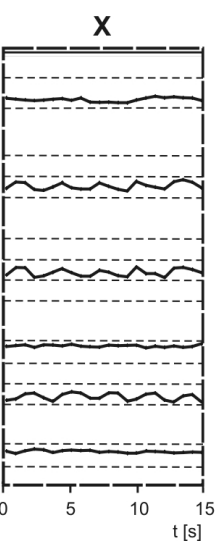
subjects.
Fig. 2. A. Continual body posturedependent beat-to-beat course of RR and of selected QRS and QRST BSPMs parameters from one subject (M.T.). Su1 - baseline, supine; TU - gradual tilting up to $+60^{\circ} ; \mathrm{Ti}$ - tilted; TD - gradual tilting down to horizontal; Su2 - supine; SU - active sitting up; Se - seated. Time scale unit - 1 minute. B. Beat-to-beat records of RR and QRST BSPMs parameters in control supine position at rest. X - selected segment in enlarged time scale, to document respiratory oscillations. In RR note the absence of sinus arrhythmia.

HF bands, was assessed with different power in all

\section{Effects of consecutive body position changes}

Basically, during body tilting and active sitting up, the character of the distributions of the respective integral maps remained analogous to that in supine position. However, as visible from the individual record (Fig. 2A), and quantified in Table 1, due to head-up tilting in the stabilized phase of the reaction, there was a highly significant decrease of the QRSTampl by $19 \%$, while of the QRSampl only by $6 \%$, and a widening of the angle $\alpha$ by $15 \%$. The NDI decreased in both the QRS and QRST BSPMs. The variability of QRSampl and of angle $\alpha$ increased substantially in the head-up position, as indicated by the increments of the respective coefficients of variation from 3.1 to $6.3 \%$ and from 4.4 to $9.1 \%$. Hemodynamically, the maximum of the reaction was characterized by a significant decrease of systolic BP by $13 \%$ (from the supine average of $130 \pm 3$ to the minimum of $113 \mathrm{~mm} \mathrm{Hg})$, mean BP by $10 \%(97 \pm 1.5$ to 


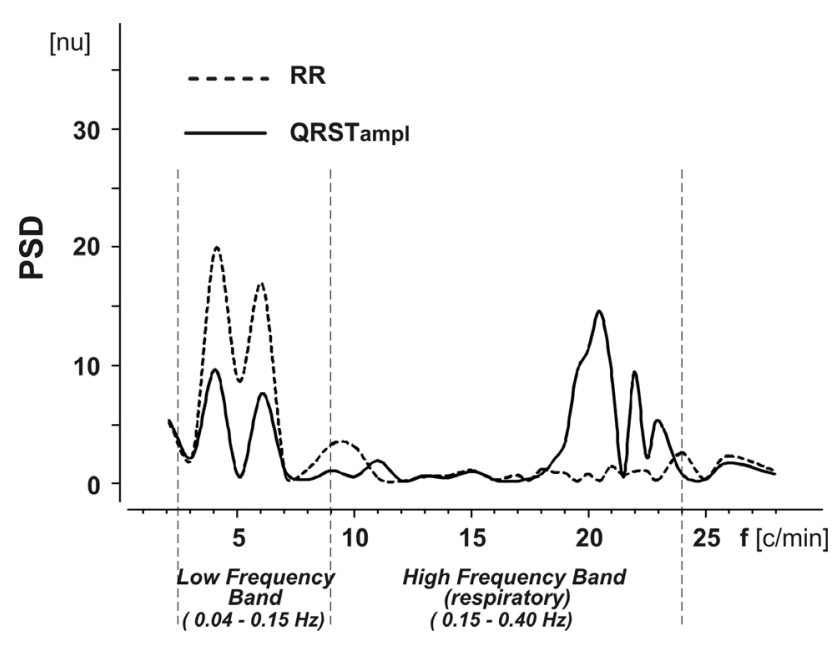

Fig. 3. Power spectra density (PSD) of simultaneous $R R$ and QRSTampl records in control supine position at rest, documenting two clearly separated low and high frequency components in QRSTampl fluctuations, however in RR variability only the low frequency component is present. nu - normalized units in \% of the respective total variance.

$87 \mathrm{~mm} \mathrm{Hg}$ ), with minimal but significant shortening of RR by $4 \%$. Consecutive tilting down to horizontal had an opposite effect, returning the QRST BSPM parameters back, almost to the control supine level.

The highly significant QRSTampl decrements in the first moments of the tilted or seated positions (by $27 \%$ or $33 \%$ respectively) were similar as compared to the preceding supine levels. However, there were postural differences in the stabilized levels of QRSTampl, which remained depressed during the whole semi-vertical tilted position, while in the seated position there was a tendency to compensate the initial postural effect (Fig. 2A, Table 1).

There are remarkable signs of dynamic transition phenomena in reaching the new postural level of the parameters investigated. Notice particularly the considerable latency and gradual return to the control supine values, which were almost reached only in the third minute of being supine after tilting down, or the long-lasting adaptation to active sitting-up (Fig. 2A).

Tilting up to a semi-vertical position caused minute inferior and left torso shifts of the distributions, approximately by $2-3 \mathrm{~cm}$. In the particular subject (M.T.), the localization of the QRSmax on the grid, changed in column 8 from row 9, to row 8, and of the QRSTmax from column 5 - row 9, to column 6 - row 8, whereby no shifts were observed in the ST distribution. Immediately after tilting down, the localization of the QRSmax and QRSTmax returned to the original position.
The postural effects on BSPMs parameters in groupmean evaluation

In spite of the substantial inter-individual variability, the highly significant body positiondependent differences of the selected representative group-mean parameters QRSTampl and QRSampl (Fig. 4) confirmed the characteristic trends of the dynamic pattern and proportions of the reactive postural depolarization and repolarization BSPMs changes, analyzed above in detail in the individual beat-to-beat sequences. With position changes from supine to $60^{\circ}$ head-up, there was a significant gradual decrease in the QRSTampl, in all subjects, in the average to $73 \%$ and with sitting to $83 \%$ of the resting supine value $(p<0.001)$ (Fig. 4). Simultaneously the angle $\alpha$ significantly $(p<0.001)$ increased in the average by $12.3^{\circ}$ in tilted and by $13.0^{\circ}$ in the seated position and the nondipolarity QRST BSPM indexes fell, to $87 \%$ and to $78 \%$ of their respective supine values $(\mathrm{p}<0.001)$. All these reactive changes were characterized by transition phenomena and prolonged after-effects (Fig. 2A, Fig. 4) Tilting back to horizontal restored the resting supine values within 3-4 min, in all subjects. The postural effects on depolarization were individually more variable and in the average head up tilting caused a minimal QRSampl increase.

The minimal systolic blood pressure during tilted position reached in the average $93 \%$ of the supine values $(p<0.05)$, but due to the increased heart rates the double products increased in the average up to $122 \%$ $(\mathrm{p}<0.01)$. On the other hand, in the seated position the systolic blood pressure in the average did not differ, while the diastolic increased up to $120 \%(\mathrm{p}<0.001)$ compared to the respective resting supine values.

\section{Discussion}

The presented qualitative and quantitative characteristics of depolarization and repolarization timeintegral BSPMs at rest are concordant with previous reports for clinically normal males (Montague et al. 1981, Sutherland et al. 1983, Green et al. 1985, Kozlíková et al. 1988, Kozmann et al. 1998, Slavíček et al. 2001, Ruttkay-Nedecký and Regecová 2002, Kellerová et al. 2006).

Circulatory adaptations, involving autonomic control, constitute an essential part of the "spontaneous" intra-individual variability of the cardiovascular functions investigated. Electrophysiological parameters characterizing ventricular repolarization have repeatedly been shown 
Table 1. Data range of selected characteristics of the integral QRS and QRST body surface potential maps in stabilized phases of different postural reactions (subject M.T.)

\begin{tabular}{|c|c|c|c|c|c|c|c|c|c|}
\hline & & \multicolumn{2}{|c|}{ supine1 $(n=400)$} & \multicolumn{2}{|c|}{ tilted $(\mathrm{n}=329)$} & \multicolumn{2}{|c|}{ supine2 $(\mathrm{n}=301)$} & \multicolumn{2}{|c|}{ sitting $(\mathrm{n}=153)$} \\
\hline & & mean & S.D. & mean & S.D. & mean & S.D. & mean & S.D. \\
\hline \multirow{4}{*}{ QRST } & $\operatorname{ampl}\left[\mu V_{s}\right]$ & 140.6 & 5.6 & $114.5^{*}$ & $5.8^{*+}$ & 134.6 & 6.7 & $128.9^{*+}$ & $4.0^{*+}$ \\
\hline & $\max [\mu \mathrm{Vs}]$ & 107.7 & 4.7 & $88.6^{*}$ & 5.1 & 102.6 & 5.6 & $90.9^{*+}$ & $3.5^{*+}$ \\
\hline & $\min [\mu V s]$ & -32.9 & 1.2 & $-25.9^{*}$ & $1.2^{*}$ & -32.0 & 1.5 & $-37.9^{*+}$ & $1.0^{*+}$ \\
\hline & $N D I[\%]$ & 16.3 & 0.6 & $13.0^{*}$ & $0.6^{*}$ & 18.0 & 1.0 & $17.8^{+}$ & $0.6^{*}$ \\
\hline \multirow{4}{*}{$Q R S$} & $\operatorname{ampl}[\mu V s]$ & 52.2 & 1.6 & $49.2^{*}$ & $3.1^{*}$ & 48.3 & 1.8 & $43.6^{*+}$ & $1.6^{+}$ \\
\hline & $\max [\mu V s]$ & 36.4 & 1.2 & 31.1* & 1.3 & 30.6 & 1.2 & $24.4^{*+}$ & $0.9^{*+}$ \\
\hline & $\min [\mu V s]$ & -17.8 & 1.2 & -18.0 & $2.3^{*}$ & -17.7 & 1.2 & $-19.2^{*+}$ & $1.3^{+}$ \\
\hline & $N D I[\%]$ & 12.2 & 0.7 & $8.0^{*}$ & $1.0^{*}$ & 12.6 & 1.1 & $14.1^{*+}$ & $0.7^{*+}$ \\
\hline$\alpha$ & [degree] & 42.9 & 1.9 & $49.5^{*}$ & $4.5^{*}$ & 47.5 & 2.6 & $52.9^{*+}$ & $3.2^{*+}$ \\
\hline$R R$ & {$[\mathrm{~ms}]$} & 737.5 & 20.6 & $712.3^{*}$ & $16.8^{*}$ & 775.1 & 25.7 & $745.1^{*+}$ & $12.2^{*+}$ \\
\hline
\end{tabular}

Data are mean and S.D. Significance of differences: bold $p<0.01$ compared with corresponding mean or S.D. values in supine1, $* p<0.01$ compared with corresponding mean or S.D. values in supine2, ${ }^{+} p<0.01$ compared with corresponding mean or S.D. values in tilted position. Numbers of evaluated successive heart beats are indicated in brackets.

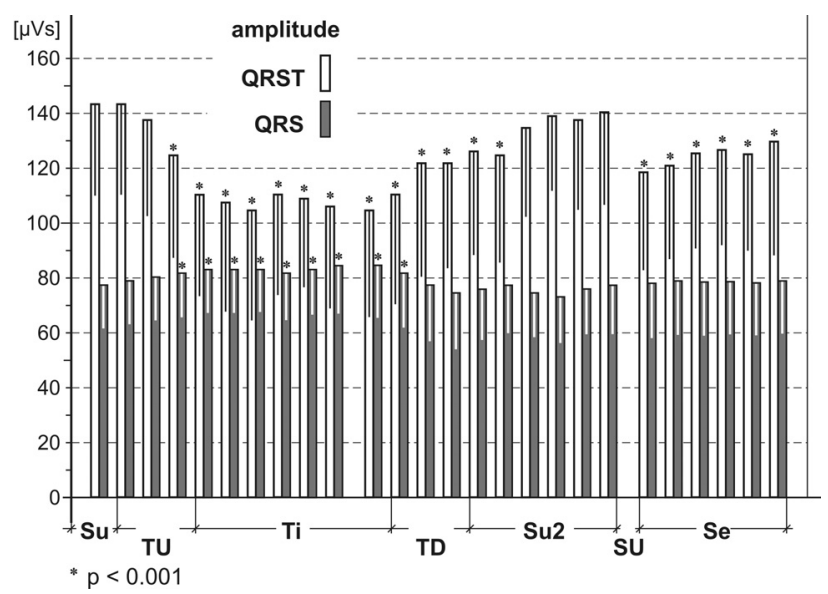

Fig. 4. Mean group values and corresponding S.D. of QRSTampl and QRSampl, averaged in $30 \mathrm{~s}$ intervals during consecutive body position changes. Su - baseline, supine; TU - gradual tilting up to $+60^{\circ} ; \mathrm{Ti}$ - tilted; TD - gradual tilting down to horizontal; Su2 - supine; SU - active sitting up; Se - seated; * significantly different in relation to respective baseline supine values.

to carry information on the direct effect of sympathetic activation on the ventricular myocardium (Ruttkay-Nedecký 2001). Takei et al. (1999) documented experimentally that sympathetic stimulation by different abbreviation of the epicardial, mid-myocardial and endocardial monophasic action potentials significantly reduced the transmural dispersion of repolarization in the canine heart in vivo. Analogical "primary" functional changes of ventricular repolarization may be produced in normal persons by a variety of physiological situations - in particular due to psychoemotional stress (Ruttkay-Nedecký 1978, Heslegrave and Furedy 1979, Contrada et al. 1989, Kellerová and Štulrajter 1991, Kellerová et al. 2006), active coping tasks to aversive stimuli (Rau 1991), smoking (Dilaveris et al. 2001), head-up position (RuttkayNedecký and Kellerová 1960, Sutherland et al. 1983, Schusterman et al. 1998, Andrásyová et al. 2001, Regecová et al. 2003), to adrenergic agonists (Kellerová et al. 1984, Contrada et al. 1989), or to the opposite effect of beta-receptor antagonists (Contrada et al. 1989, Rau 1991). A characteristic pattern of repolarization parameters, pointing to an increased sympathetic drive of the ventricular myocardium, was described not only in hypertension (Kellerová et al. 1999, Regecová et al. 2000, Andrásyová et al. 2001, Regecová et al. 2003) but also in subjects just exceeding the casual blood pressure value of 120/80 mm Hg (Kellerová and Regecová 2009), defined as a limit between normal and prehypertensive BP.

In earlier studies, the effect of factors increasing the sympathetic drive of the heart, and thus influencing its electrical activity, namely the pattern of ventricular repolarization, was assessed by changes in T-wave shape and decrease of its amplitude (Ruttkay-Nedecký 1978, Guazzi et al. 1978, Heslegrave and Furedy 1979, Contrada et al. 1989, Ruttkay-Nedecký 2001). To enable 
quantitative evaluation of the functional changes in repolarization, the maximal spatial T-vector was proposed as a convenient parameter (Ruttkay-Nedecký 1978). In VCG studies, an increased sympathetic outflow to the ventricular myocardium was documented by the decreased magnitude of the maximal spatial repolarization vector sTmax and of the ventricular gradient, as well as by an increased spatial angle between QRS and STT area vectors, reflecting a decrease of the repolarization heterogeneity in the ventricular myocardium (Ruttkay-Nedecký 1978, Kellerová and Štulrajter 1991, Dilaveris et al. 2000, Pišvejcová et al. 2002, Regecová et al. 2003). With the progress in computer processing of BSPM data, it has been suggested that non-uniform or disparate ventricular recovery properties are direct determinants of the QRST deflection area and of an increased non-dipolar content of QRST BSPM (Sutherland et al. 1983, Abildskov and Green 1987). All these studies were based on a static, mainly group-mean evaluation of the effect of the described interventions.

The reactive changes in the BSPM characteristics of cardiac repolarization, provoked by a tilt table test and by sitting up recorded in the present study, namely the decrease of the QRSTampl, the decrease of the QRST BSPM nondipolarity index and an increased angle $\alpha$ between QRS and QRST eigenvectors, are in general agreement with others, describing by group-mean values after head-up tilt or active standing, a $24 \%$ decline of the peak T wave amplitude (Schusterman et al. 1998), significant shortening of the ventricular gradient (Ruttkay-Nedecký and Kellerová 1960), $15-31 \%$ decrease of the maximal spatial $\mathrm{T}$ vector (Andrásyová et al. 2001, Regecová et al. 2003), decrease of BSPM ST maximum by 17 \% (Sutherland et al. 1983). However, the present study is the first to show the dynamics in the course of these reactive postural changes in the body surface ECG pattern. There is a considerable latency in reaching the new, stabilized, postural level of the BSPM parameters in the tilted position and in their return to the control supine values. The muscular activity and the intermediate level of gravitational stimulation induced by active sitting, probably account for cardiovascular adaptation and - in spite of the seated position - for the gradual return of the altered repolarization parameters to their control levels.

The effect of changing body posture on the repolarization BSPM may be considered first of all as a result of an improved homogeneity of ventricular recovery, indicating the effect of an increased sympathetic drive of the heart (Takei et al. 1999). Due to the gravitational stress (tilt, sitting up), when in the first moments blood pooling in the lower part of the body reduces venous return and cardiac output, causing the arterial underfilling, the sympathetic nervous system is activated to keep the arterial pressure at optimal level. This explanation is supported also by typical transition phenomena in reaching the new postural level of BSPMs parameters and prolonged after-effects, documented both by the individual as well as group evaluations, after tilting maneuvers or sitting up.

The positional change of the heart in relation to the recording electrodes was supposed to be another possible underlying cause of the postural BSPM differences. Some minimal inferior torso shifts of the distributions were detectable in the depolarization QRS BSPM (in the chest surface grid points display by one row downwards), contributing also to the shift of the QRST BSPM, while the distribution of the repolarization ST BSPM remained spatially unaltered. On the other hand, from supine to upright position, there was a highly significant decrease of the QRSTampl and of the derived STampl in all subjects investigated, with minimal and individually variable change of the QRSampl. The same holds true for maxima of BSPMs, both located on the anterior chest, which are not affected equally. In the average there was a significant decrement of QRST BSPM max by $24 \%$ but that of QRS BSPM max only by $14 \%$ and an increase of QRS BSPM min by $25 \%$. These results suggest that some other factors had to be operative at the same time, as one would reasonably expect a comparable effect of the postural changes in heart-tobody surface relation on depolarization as well as repolarization BSPMs parameters. In contrast to cardiac depolarization, characterized by wave-front propagation, which may be more sensitive to the body posture associated effects resulting in QRS distribution shifts, the widely spatially and temporally spread myocardial repolarization processes may be more sensitive to subtle changes in the neurally or humorally mediated interactions at the membranes of the cardiac effector cells, induced by sympathetic activation and ultimately resulting in a highly significant decrease of the QRST and ST amplitudes. These premises are supported by previously described orthostatic shortening of the ventricular gradient (Ruttkay-Nedecký and Kellerová 1960) or significant diminution of the maximal spatial repolarization vector (sTmax), computed from the 
corrected orthogonal leads and not influenced by changes of the heart to body position (Andrásyová et al. 2001).

This study is the first to report on the chronological course and intra-individual variability of spontaneous or reactive BSPM changes in a dynamic way, from beat-to-beat. To our knowledge, no information has been reported on spectral analysis of the cardiac electric field variability, capable of assessing dynamic changes in the autonomic control of ventricular recovery. Even during normal tidal respiration, at rest with a frequency of $15-20 / \mathrm{min}$, respiration-related oscillations were visible in all records of BSPM parameters. They were not solely heart rate-dependent, as they were significantly present in some subjects also in the absence of sinus arrhythmia in the RR interval series. Respiratory sinus arrhythmia is generally accepted as a reliable cardiac index for estimating the level of vagal cardiac nerve activity. However, recent findings suggest that suppression of respiratory sinus arrhythmia by sympathetic effects on the sinoatrial node can be substantial. Striking is the wide intersubject difference of this sympathetic effect (Taylor et al. 2001). Quiet respiration is associated with parallel phasic changes in the activity of spinal muscle sympathetic motonuclei in man. It has been suggested that the same events that relate to a burst of sympathetic impulses in muscle nerves also change cardiac autonomic outflow (Wallin and Nerhed 1982). Besides the respiratory periodicities in sympathetic activity, there are other factors, like rotation of the heart, blood $\mathrm{pCO}_{2}, \mathrm{pO}_{2}$ and $\mathrm{pH}$, insulating effect of the inflated lungs, oscillations in the venous return, etc., which may contribute to the respiratory oscillation in ventricular repolarization.

Periodic variations in the repolarization BSPM parameters in the low-frequency band, visible in the individual records by the naked eye and preliminarily evaluated by the Discrete Fourier Transform frequency analysis of the QRSTampl as well as of RR, are similar to the many times described LF variations in blood pressure and in vasomotor activity (Stauss 2003). This finding will be a subject of a more detailed forthcoming study. However, we support the hypothesis, that LF component of BSPMs variability constitutes a marker of oscillations in the sympathetic drive of ventricles. "While the ratio between LF a HF frequency powers in the RR interval time series analysis may be of value in characterizing a certain state of the autonomic regulation of the pacemaker, it cannot give pertinent information on the state of the autonomic regulation of the cardiac ventricles" (Ruttkay-Nedecký 2001). In this respect, the spectral analysis of the ventricular repolarization pattern in the BSPM is promising for recognizing spontaneous oscillations of the sympathetic drive of the ventricles.

\section{Conclusions}

This study based on beat-to-beat analysis of a train of ECG body surface potential maps provided the first demonstration of spontaneous, non-random, respiratory and low-frequency oscillations of the ventricular repolarization pattern, and the first insight into the dynamics of body posture-associated changes in ventricular activation and recovery.

\section{Conflict of Interest}

There is no conflict of interest.

\section{Acknowledgements}

The authors wish to express their gratitude to Prof. R. L. Lux, CVRTI, University of Utah, Salt Lake City for the "transfer matrix" kindly provided to Prof. G. Kozmann, and used in this study, and to Dr. V. Regecová for the somatometric measurements. This work was supported by the Slovak Scientific Grant Agency (VEGA No. 2/6187/28) and by grants TÁMOP-4.2.2-08/1/2008-0018 and Tech_08_D3 AALAMSRK of the National Office of Research and Development, Budapest, Hungary.

\section{References}

ABILDSKOV JA, GREEN LS: The recognition of arrhythmia vulnerability by body surface electrocardiographic mapping. Circulation 75 (Suppl III): 79-83, 1987.

ABILDSKOV JA, GREEN LS, LUX RL: Detection of disparate repolarization by means of the body surface electrocardiogram. In: Cardiac Electrophysiology and Arrhythmias. DP ZIPES, J JALIFE (eds), Grune\&Stratton, Orlando, 1985, pp 495-499. 
ANDRÁSYOVÁ D, REGECOVÁ V, TONKOVIČ M, KELLEROVÁ E, KRČ-TURBOVÁ Z, NOVOTNÁ E: Sensitive markers of the repolarization alterations in systemic hypertension. Bratisl Lek Listy 102: 530-535, 2001.

CONTRADA RJ, KRANTZ DW, DUREL LA, LEVY L, LAROCCIA PL, ANDERSON JR, WEISS T: Effects of betaadrenergic activity on T-wave amplitude. Psychophysiology 26: 488-492, 1989.

DILAVERIS P, GIALAFOS E, POLONIECKI J, HNATKOVA K, RICHTER D, ANDRIKOPOULOS G, LAZAKI E, GIALAFOS J, MALIK M: Changes of the T wave amplitude and angle: an early marker of altered ventricular repolarization in hypertension. Clin Cardiol 23: 600-606, 2000.

DILAVERIS P, PANTAZIS A, GIALAFOS E, TRIPOSKIADIS F, GIALAFOS J: The effect of cigarette smoking on the heterogeneity of ventricular repolarization. Am Heart J 142: 833-837, 2001.

GREEN LS, LUX RL, HAWS CW, WILLIAMS RL, HUNT SC, BURGESS MJ: Effects of age, sex and body habitus on QRS and ST-T potential maps of 1100 normal subjects. Circulation 71: 244-253, 1985.

GUAZZI MD, MAGRINI F, OLIVARI MT, POLESE A, FIORENTINI C: Influence of the adrenergic nervous system on the repolarization phase of the electrocardiogram. Angiology 29: 617-630, 1978.

HESLEGRAVE RJ, FUREDY JJ: Sensitivities of heart rate and $\mathrm{T}$ wave amplitude for detecting cognitive and anticipatory stress. Physiol Behav 22: 17-23, 1979.

HOEKEMA R, UIJEN GJ, STILLI D, VAN OSTEROM A: Lead system transformation of body surface map data. J Electrocardiol 31: 71-82, 1998.

KELLEROVÁ E, REGECOVÁ V: Repolarization pattern indicates enhanced sympathetic drive of the ventricular myocardium already at normal and high-normal blood pressure. Kidney Blood Press Res 32: 311, 2009.

KELLEROVÁ E, ŠTULRAJTER V: Different effect of the adrenergic neurohumoral activation caused by psychoemotional stress on the repolarization part of the ECG in trained sportsmen. Acta Fac Educ Phys Univ Com 30: 113-118, 1991.

KELLEROVÁ E, VIGAŠ M, KVETŇANSKÝ R, JEŽOVÁ D: The influence of dopamine on the maximal spatial repolarization vector of the human heart. In: Electrocardiology '83. I RUTTKAY-NEDECKÝ, P MACFARLANE (eds), Excerpta Medica, Amsterdam, 1984, pp 78- 83.

KELLEROVÁ E, REGECOVÁ V, ANDRÁSYOVÁ D: Evidence for increased vasomotor and myocardial sympathetic drive in hypertensives and subjects with „high normal“ blood pressure. Cor Vasa 41: K 150, 1999.

KELLEROVÁ E, REGECOVÁ V, KATINA S, TITOMIR LI, AIDU EAI, TRUNOV VG, SZATHMÁRY V: The effect of psychoemotional load on ventricular repolarization reflected in integral body surface potential maps. Physiol Res 55 (Suppl 1): 99-105, 2006.

KHADDOUMI B, RIX H, MESTE O, FERENIEC M, MANIEWSKI R: Body surface ECG signal shape dispersion IEEE Trans Biomed Eng 53: 2491-2500, 2006.

KOZLÍKOVÁ K, TURZOVÁ M, TYŠLER M, POPPEROVÁ E, SABOLOVÁ K, PETRÁŠOVÁ H, MICHALÍK D: Body surface integral mapping of ventricular activation in boys during puberty. Bratisl Lek Listy 89: 694-705, 1988.

KOZMANN GY: Beat-to-beat dynamics of ventricular depolarization and repolarization. Cardiology 15: 21S, 2006. (Abstract)

KOZMANN G, FARKAS N, SANDOR G, SZAKOLACZAI K, SZATHMARY V, TYSLER M, TURZOVA M: QRST integral maps of normal subjects. Statistical analysis and simulation study. Comput Cardiol 25: 521-524, 1998.

KOZMANN GY, HARASZTI K: Importance of body surface potential field representation fidelity: analysis of beat-tobeat repolarization measurements. Anadolu Kardiyol Der 7 (Suppl 1): 5-7, 2007.

LUX RL, SMITH CR, WYATT RF, ABILDSKOV JA: Limited lead selection for estimation of body surface potential maps in electrocardiography. IEEE Trans Biomed Eng 25: 270-276, 1978.

LUX RL, EVANS AK, BURGESS MJ, WYATT RF, ABILDSKOV JA: Redundancy reduction for improved display and analysis of body surface potential maps. 1. Spatial compression. Circ Res 49: 186-196, 1981.

MONTAGUE TJ, SMITH ER, CAMERON DA, RAUTAHARJU PM, KLASSEN GA, FLEMINGTON CS, HORACEK BM: Isointegral analysis of body surface maps: Surface distribution and temporal variability in normal subjects. Circulation 63: 1166-1172, 1981. 
PIŠVEJCOVÁ K, PACLT I, SLAVÍČEK J, KITTNAR O, DOHNALOVÁ A, KITZLEROVÁ A: Electrocardiogram, vectorcardiogram and body surface maps in patients with panic disorder. Physiol Res 51: 401- 406, 2002.

RAU H: Response of the T-wave amplitude as a function of active and passive tasks and beta-adrenergic blockade. Psychophysiology 28: 231-239, 1991.

REGECOVÁ V, ANDRÁSYOVÁ D, RUTTKAY-NEDECKÝ I: Electrocardiologic objectivisation of sympathetic nervous drive of ventricles in subjects with elevated blood pressure and in sportsmen. Homeostasis Hlth Dis 41: 118-120, 2000.

REGECOVÁ V, KELLEROVÁ E, ANDRÁSYOVÁ D: Influence of the body position on the maximal special T-vector in boys and young men with increased diastolic blood pressure. Physiol Res 52: 38, 2003. (Abstract)

RUTTKAY-NEDECKÝ I: Effect of emotional stress on cardiac repolarization vectors. Adv Cardiol 21: 284-285, 1978.

RUTTKAY-NEDECKÝ I: The effect of the autonomic nervous system on the heart. Electrocardiographic evaluation: Problems and concerns. Cardiology 10: 42-48, 2001.

RUTTKAY-NEDECKÝ I, KELLEROVÁ E: Ortostatic changes of the ventricular gradient in electrocardiograms (in Slovak). Bratisl Lek Listy 40: 513-522, 1960.

RUTTKAY-NEDECKÝ I, REGECOVÁ: Normal variability of the peak-to through amplitude of isointegral QRST body surface maps. $J$ Electrocardiol 35: 327-332, 2002.

SCHUSTERMAN V, AYSIN B, SHAH I, FLANIGAN S, ANDERSON KP: Autonomic nervous system effects on ventricular repolarization and RR interval variability during head-up tilt. Comput Cardiol 25: 717-720, 1998.

SLAVÍČEK J, PACLT I, HAMPLOVÁ J, KITTNAR O, TREFNÝ Z, HORÁČEK BM: Antidepressant drugs and heart electrical field. Physiol Res 47: 297-300, 1998.

SLAVÍČEK J, KITTNAR O, TREFNÝ Z, HORÁČEK BM: ECG body surface isointegral and isoarea maps (BSM) in 30- and 60-year-old healthy humans. Sbor lék 102: 369-374, 2001.

STAUSS HM: Heart rate variability. Am J Physiol 285: R927-R931, 2003.

SUTHERLAND DJ, MCPHERSON DD, SPENCER CA, ARMSTRONG CS, HORACEK BM, MONTAGUE TJ: Effects of posture and respiration on body surface electrocardiogram. Am J Cardiol 52: 595- 600, 1983.

TAKEI M, YASUYUKI S, TAKANORI Y, MANOI L, MASAKAZU A, KENDO K: The autonomic control of the transmural dispersion of ventricular repolarization in anesthetized dogs. J Cardiovasc Electr 10: 981-989, 1999.

TAYLOR JA, MYERS CHW, HALLIWILL JR, SEIDEL H, ECKBERG DL: Sympathetic restraint of respiratory sinus arrhythmia: implications for vagal-cardiac tone assessment in humans. Am J Physiol 280: H2804-H2814, 2001.

WALLIN BG, NERHED C: Relationship between spontaneous variations of muscle sympathetic nerve activity and succeeding changes of blood pressure in man. J Auton Nerv Syst 6: 293-302, 1982.

ŽĎÁRSKÁ D, PELÍŠKOVÁ P, CHARVÁT J, SLAVÍČEK J, MLČEK M, MEDOVÁ E, KITTNAR O: ECG body surface mapping (BSM) in Type 1 diabetic patients. Physiol Res 56: 403-410, 2006. 